\title{
A distinct metabolic response characterizes sensitivity to EZH2 inhibition in multiple myeloma
}

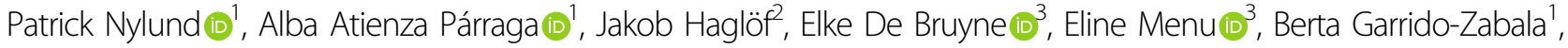
Anqi $\mathrm{Ma}^{4}$, Jian Jin (1) ${ }^{4}$, Fredrik Öberg' ${ }^{1}$, Karin Vanderkerken ${ }^{3}$, Antonia Kalushkova (D) ${ }^{1}$ and Helena Jernberg-Wiklund (1) ${ }^{1}$

\begin{abstract}
Multiple myeloma (MM) is a heterogeneous haematological disease that remains clinically challenging. Increased activity of the epigenetic silencer EZH2 is a common feature in patients with poor prognosis. Previous findings have demonstrated that metabolic profiles can be sensitive markers for response to treatment in cancer. While EZH2 inhibition (EZHZi) has proven efficient in inducing cell death in a number of human MM cell lines, we hereby identified a subset of cell lines that despite a global loss of H3K27me3, remains viable after EZH2i. By coupling liquid chromatography-mass spectrometry with gene and miRNA expression profiling, we found that sensitivity to EZH2i correlated with distinct metabolic signatures resulting from a dysregulation of genes involved in methionine cycling. Specifically, EZH2i resulted in a miRNA-mediated downregulation of methionine cycling-associated genes in responsive cells. This induced metabolite accumulation and DNA damage, leading to G2 arrest and apoptosis. Altogether, we unveiled that sensitivity to EZH2i in human MM cell lines is associated with a specific metabolic and gene expression profile post-treatment.
\end{abstract}

\section{Introduction}

Multiple myeloma (MM) is a genetically and clinically heterogeneous haematological disease, characterized by a monoclonal expansion of malignant plasmablasts/plasma cells in the bone marrow ${ }^{1-3}$. A complex mutational landscape, high heterogeneity and a genome-wide epigenetic reconfiguration may contribute to the underlying causes of drug resistance and relapse ${ }^{4-6}$.

We have previously demonstrated that the gene silencing profile of MM cells is reminiscent of the one of embryonic fibroblasts, where the Polycomb repressive complex 2 (PRC2) is highly active ${ }^{7}$. Moreover, an increased deposition of histone $\mathrm{H} 3$ lysine 27

\footnotetext{
Correspondence: Antonia Kalushkova (antonia.kalushkova@igp.uu.se) or Helena Jernberg-Wiklund (helena.jernberg_wiklund@igp.uu.se)

${ }^{1}$ Science for Life Laboratory, Department of Immunology, Genetics and Pathology, Rudbeck Laboratory, Uppsala University, Uppsala, Sweden

2Department of Medicinal Chemistry, Analytical Pharmaceutical Chemistry, Uppsala University, Uppsala, Sweden

Full list of author information is available at the end of the article These authors contributed equally: Patrick Nylund, Alba Atienza Párraga Edited by B. Zhivotovsky
}

trimethylation (H3K27me3) by PRC2 correlates with advanced stages of the disease ${ }^{4}$ and overexpression of the catalytic subunit of PRC2, enhancer of zeste homologue 2 (EZH2), is a common feature of $\mathrm{MM}^{8,9}$. These data support the notion that PRC2-mediated silencing is a key mechanism in $\mathrm{MM}$ and may represent a suitable drug target $^{4,10}$. In line with this, selective inhibition of EZH2 (EZH2i) with UNC1999 impaired cell viability and induced apoptosis in MM cell lines and primary patient samples ${ }^{11}$, and upregulated tumour suppressor miRNAs that repress oncogenes with a vital function for MM cell growth and survival $^{11-13}$.

Metabolic profiles are altered upon drug treatment, thus panels of metabolites have been used as biomarkers for drug response ${ }^{14,15}$. The association between the metabolome and the epigenome is, however, complex. It is well known that chromatin regulators require metabolite intermediates as cofactors and that different metabolic pathways associated with tumorigenesis are epigenetically regulated $^{16}$. However, the impact of epigenetic targeting on the cellular metabolome is largely unexplored.

\section{(c) The Author(s) 2021}

(c) (i) Open Access This article is licensed under a Creative Commons Attribution 4.0 International License, which permits use, sharing, adaptation, distribution and reproduction cc) in any medium or format, as long as you give appropriate credit to the original author(s) and the source, provide a link to the Creative Commons license, and indicate if changes were made. The images or other third party material in this article are included in the article's Creative Commons license, unless indicated otherwise in a credit line to the material. If material is not included in the article's Creative Commons license and your intended use is not permitted by statutory regulation or exceeds the permitted use, you will need to obtain permission directly from the copyright holder. To view a copy of this license, visit http://creativecommons.org/licenses/by/4.0/. 
In this study, we found that in vivo EZH2i with UNC1999 effectively reduced tumour load in the 5T33MM syngeneic murine model, further emphasising the potential of EZH2 as a target for clinical intervention. However, when exploring the effects of EZH2i in an extended panel of human MM cell lines, we observed that a subset of cell lines remained viable after the global loss of H3K27me3. Combining liquid chromatography-mass spectrometry (LC-MS), chromatin immunoprecipitation (ChIP), gene and miRNA expression profiling, we identified distinct alterations in the methionine cycling pathways in cell lines that responded to EZH2i, which were absent in non-responsive cell lines. These metabolic alterations e.g., homocysteine and 5-methyltetrahydrofolic acid accumulation, correlated with the upregulation of EZH2-targeted miRNAs (e.g., miR-4943p, miR-130a-3p, miR-134-5p and miR-192-5p), which in turn downregulated genes involved in the methionine and homocysteine degradation pathways (e.g., $M A T 2 A / 2 B, C B S$ and $C T H$ ). This was associated with DNA double-strand breaks, G2 arrest and apoptosis. Altogether, our study unveils a mechanism of clinical relevance that underlies responsiveness to EZH2i in human MM cell lines.

\section{Results}

UNC1999 reduced tumour burden in the 5T33MM murine model

To test the effects of EZH2i in vivo, we administered the EZH2 inhibitor UNC1999 to 5T33MM mice, which share characteristics with human $\mathrm{MM}^{17,18}$. C57BL/KaLwRij ${ }^{19}$ mice were injected with 5T33MM cells and treated with UNC1999. To ensure on-target effects of UNC1999 in vivo, we analysed H3K27me3 levels in bone marrow plasma cells from 5T33MM mice treated with $150 \mathrm{mg} / \mathrm{kg}$ and $300 \mathrm{mg} / \mathrm{kg}$ UNC1999 for seven days. We found a global reduction of H3K27me3 in UNC1999-treated mice, which was not due to a loss of total histone $\mathrm{H} 3$ (Fig. 1a-c), suggesting that UNC1999 effectively inhibited EZH2 in the bone marrow microenvironment. To evaluate its effects on the tumour burden in vivo, mice were treated with $200 \mathrm{mg} / \mathrm{kg}$ of UNC1999 or vehicle until the control group indicated signs of morbidity. We observed a decrease in bone marrow plasmacytosis, spleen weight ${ }^{20}$ and $M$-spike levels in the serum of treated mice (Fig. 1d-f). Taken together, we observed a positive treatment response to UNC1999 in the 5T33MM
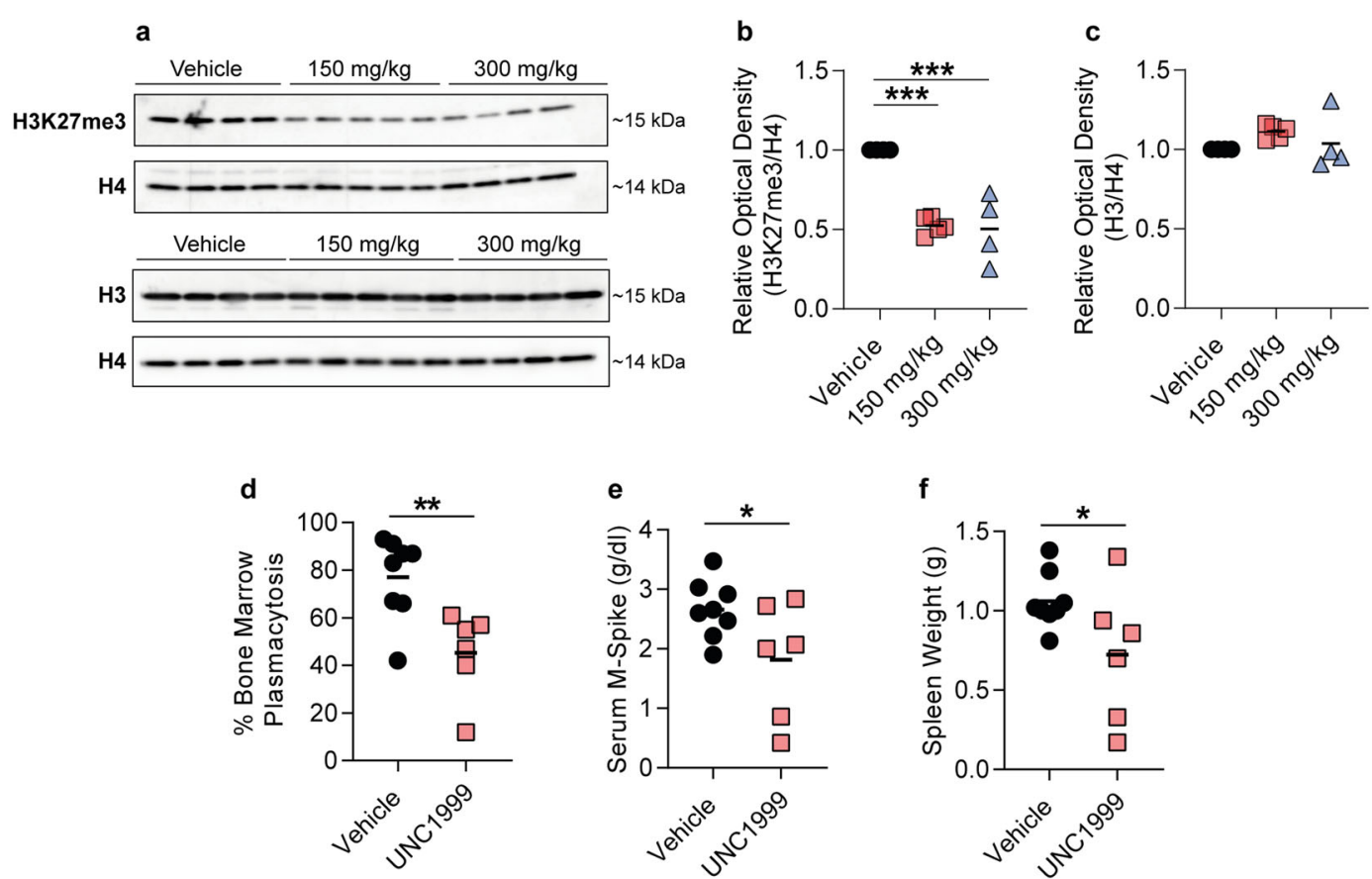

Fig. 1 EZH2 inhibition significantly reduced tumour burden in mice bearing 5T33MM multiple myeloma. a Western blot against H3K27me3 and total histone $\mathrm{H} 3(\mathrm{H} 3)$ of bone marrow plasma cells derived from 5T33MM mice treated with 150 or $300 \mathrm{mg} / \mathrm{kg}$ of UNC1999, or with vehicle. Total histone $\mathrm{H} 4(\mathrm{H} 4)$ was used as a loading control. Corresponding uncropped western blots can be found in Supplementary Fig. 9a-b. b Signal quantification of the western blot against H3K27me3 shown in (a). c Signal quantification of the western blot against H3 shown in (a). The optical density in (b-c) was normalized against H4. $n_{\text {(vehicle) }}=4, n_{(150 \mathrm{mg} / \mathrm{kg})}=5$ and $n_{(300 \mathrm{mg} / \mathrm{kg})}=4$. Statistical analysis in (b-c) was performed with one-way ANOVA. Values: mean with SEM. d-f 5T33MM mice were treated with $200 \mathrm{mg} / \mathrm{kg}$ of UNC1999 or with vehicle. Tumour load was determined by $\mathbf{d}$ bone marrow plasmacytosis, e M-spike levels and $\mathbf{f}$ spleen weight. $n_{\text {(vehicle) }}=8, n_{\text {(UNC1999) }}=6$. Statistical analysis in (d- $\mathbf{f}$ ) was performed with one-sided $t$-test. Values: mean with SEM. ${ }^{*} p<0.05,{ }^{* *} p<0.01,{ }^{* * *} p<0.001,{ }^{* * * *} p<0.0001$. 
preclinical murine model, which encouraged further investigation into an extended panel of human MM cell lines.

\section{$\mathrm{EZH} 2 \mathrm{i}$ induced a distinct metabolic signature in responsive MM cell lines}

We further assessed the response to EZH2i in seven human MM cell lines. Cells were treated with $1 \mu \mathrm{M}$ of UNC1999 for five days and cell viability was determined at the experimental endpoint. U1996, U266-1970 and KMS$28 \mathrm{PE}$ exhibited a non-responsive profile (viability at endpoint > 80\%), whereas OPM2, L363, LP-1, and INA-6 exhibited a responsive profile ( $<\sim 80 \%$ viability) (Fig. $2 \mathrm{a}$ and Supplementary Fig. 1a-d).

To investigate the underlying molecular mechanisms in responsive versus non-responsive cells after UNC1999 treatment, all cell lines were subjected to a global metabolic analysis by high-resolution LC-MS after UNC1999 or DMSO treatment. UNC1999 altered the metabolic profile in responsive cell lines to a larger extent than in non-responsive cell lines (Supplementary Fig. 1e-f). Next, we identified differentially abundant metabolites after treatment in responsive (OPM2, L363, LP-1, and INA-6) and non-responsive (U1996, KMS-28PE and U266-1970) cell lines and selected the metabolites with the highest discriminating power for further analyses (Supplementary Fig. 1g). Unsupervised hierarchical clustering based on the identified metabolites revealed that the responsive cell lines OPM2, L363 and LP-1 shared a metabolic signature under basal conditions, which was different from the one of the non-responsive cell lines KMS-28PE and U2661970 (Fig. 2b). Interestingly, despite having differential viability responses to UNC1999 treatment, U1996 and INA-6 shared a common metabolic profile under basal conditions (Fig. 2b) and EZH2i effectively reduced H3K27me3 levels in both cell lines (Fig. 2c, d). Thus, INA6 and U1996 were selected for in depth evaluation of changes in metabolite abundance after EZH2i. Principal component analysis (PCA) showed a clear discrimination between INA-6 and U1996 cells upon UNC1999 treatment (Fig. 2e). Based on the top 150 differentially abundant metabolites, a significantly altered metabolic response was observed in INA- 6 when treated with UNC1999, while the metabolic profile of U1996 remained unaffected (Fig. 2f). Similar to INA-6, the other responsive cell lines (LP-1, L363 and OPM2) showed a shift in metabolic response as a result of EZH2i (Supplementary Fig. 2a-d). In summary, we identified that sensitivity to EZH2i was associated with altered metabolic response.

\section{Sensitivity to EZH2i was associated with alterations in the methionine cycling pathways}

We next used MetaboAnalyst ${ }^{21}$ to decipher which cellular pathways were involved in the metabolic shift observed in responsive MM cell lines post-UNC1999 treatment. Metabolites highly enriched after UNC1999 treatment were examined by metabolite set enrichment analysis (MSEA). The analysis unveiled a dysregulation of pathways linked to methionine cycling (e.g., betaine metabolism and methionine metabolism) in INA-6 (Fig. 2g), similarly to what was observed for the other responsive cell lines (Supplementary Fig. 2e-g). Due to a lack of sufficient discriminating features in 2/3 resistant cell lines (U266-1970 and U1996), it was not possible to generate individual models, suggesting that metabolic changes induced by EZH2i are associated with sensitivity. Thus, we generated a model that merged the resistant cell lines and found that the only significantly deregulated pathway was homocysteine degradation (Fig. 2h). Importantly, we did not detect major alterations in housekeeping molecules in INA-6 or U1996 cells, suggesting an intact cellular integrity (Supplementary Table I).

On a metabolite level, UNC1999-treated INA-6 cells showed an accumulation of 5-methyltetrahydrofolic acid (Fig. 3a), a decrease in glycine abundance (Fig. 3b) and an accumulation of 5-methylthioadenosine (Fig. 3c), while methionine and adenosine remained unaffected (Supplementary Fig. 3j-k). Similar effects were observed in one or more of the other responsive cell lines (Supplementary Fig. 3a-c, e-f). Interestingly, we found a clear accumulation of homocysteine in INA-6 cells after UNC1999 treatment, which was 6.8 times larger than in the DMSOtreated control (Fig. 3d), while the by-products of homocysteine degradation showed no alterations (Supplementary Fig. 3l-n). The other responsive cell lines showed variable responses in terms of accumulation of metabolites involved in homocysteine degradation (Supplementary Fig. 3d, h-i). No significant changes in specific metabolites were observed in the non-responsive cell lines (Fig. $3 g-j$ and Supplementary Fig. 3a-i). Altogether, sensitivity to EZH2i was associated with changes in the abundance of metabolites within the methionine cycling pathways.

\section{Sensitivity to EZH2i correlated with downregulation of genes involved in methionine cycling}

We then sought to investigate whether the alterations in metabolite abundance were associated with changes in the expression of genes involved in methionine cycling. UNC1999-treated INA-6 cells downregulated genes involved in methionine metabolism such as methionine adenosyltransferase 2A $(M A T 2 A)$, methionine adenosyltransferase 2B (MAT2B) and adenosyl-homocysteinase $(A H C Y)$. In addition, cystathionine-beta-synthase (CBS) and cystathionase $(C T H)$ in the homocysteine degradation pathway decreased in expression post-treatment (Fig. 3e-i), in line with the observed accumulation of homocysteine 
a

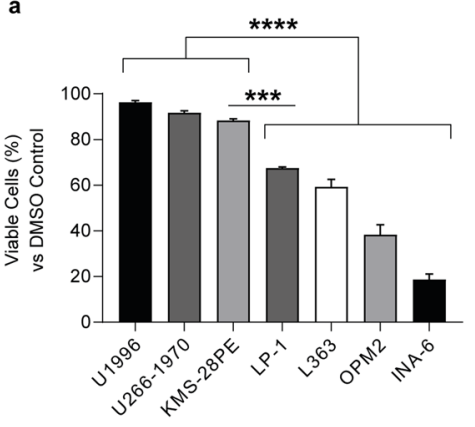

c
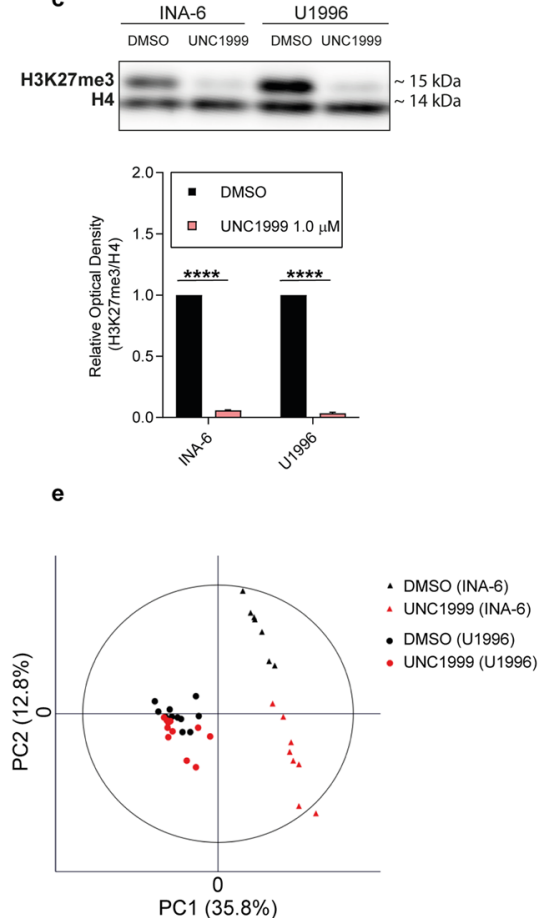

g

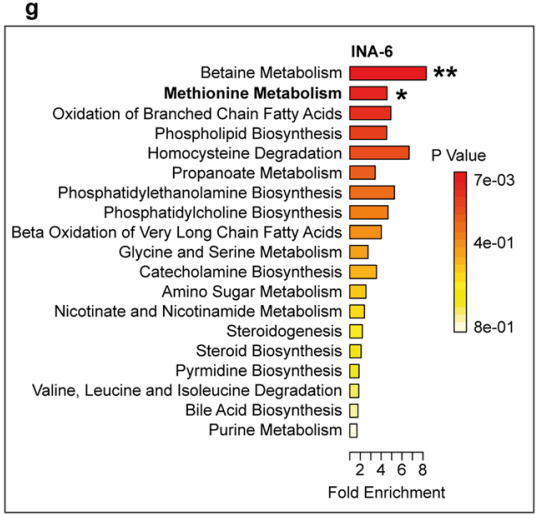

b

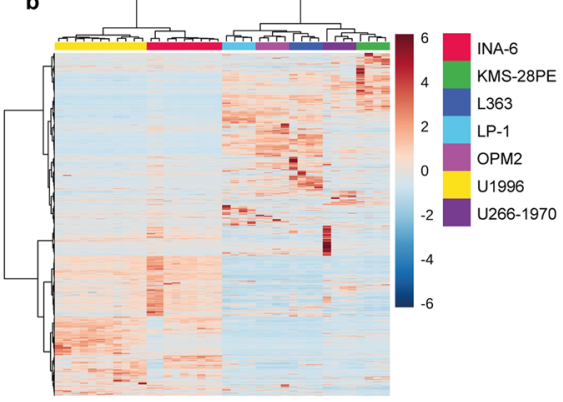

d
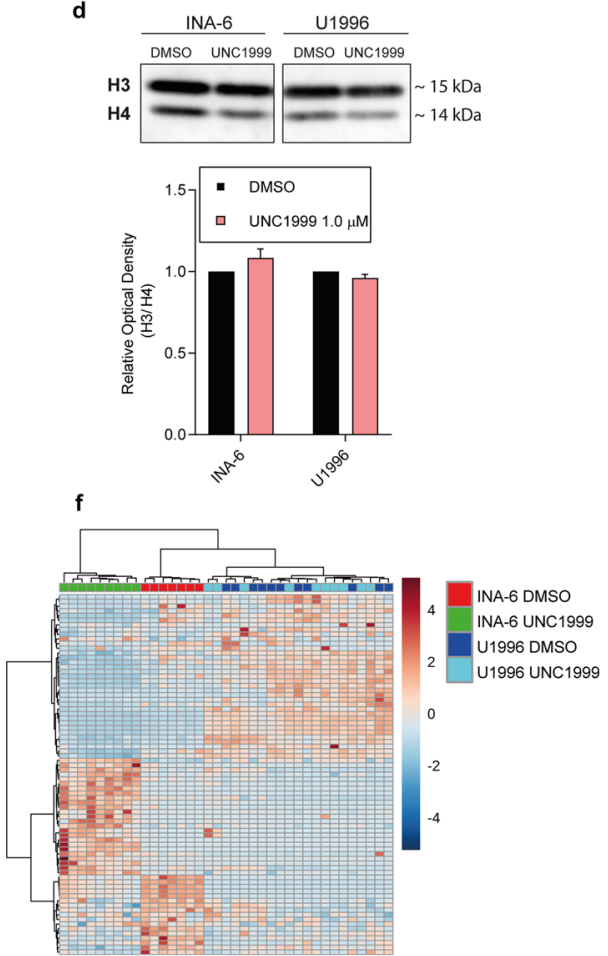

h

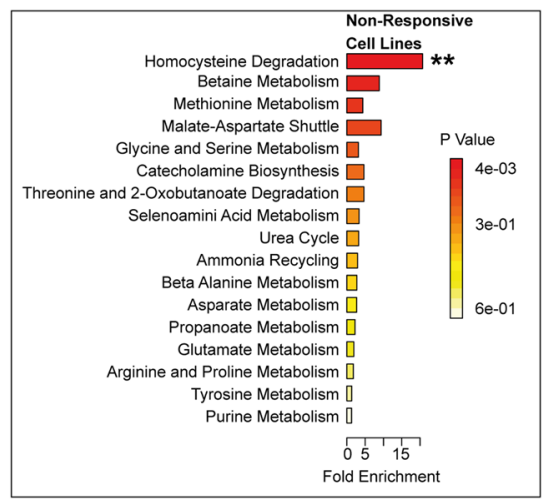

Fig. 2 (See legend on next page.)

(Fig. 3d). Consistent with a reduced glycine abundance and the accumulation of 5-methyltetrahydrofolic acid (Fig. 3a-b), UNC1999 treatment also decreased the expression of 5-methyltetrahydrofolate-homocysteinemethyltransferase (MTR), methylenetetrahydrofolate reductase (MTHFR) and serinehydroymethyltransferase 2 (SHMT2) (Fig. 3j-1). 
(see figure on previous page)

Fig. 2 Sensitivity of MM cell lines to UNC1999 treatment was associated with metabolic alterations in methionine metabolism. a Effects of UNC1999 treatment on cell viability for the indicated cell lines. Biological replicates $=3$. Statistical analysis was performed with one-way ANOVA. Values: mean with SEM. $\mathbf{b}$ Unsupervised clustering of seven human MM cell lines based on the abundance of all metabolites detected by LC-MS under basal conditions (DMSO treatment only). $n_{(\text {INA-6) }}=9, n_{(\mathrm{U} 1996)}=11, n_{(\mathrm{KMS}-28 \mathrm{PE}, \text { U266-1970, LP-1, L363 and OPM2) }}=4$. c Representative western blot against H3K27me3 in the INA-6 (responsive) and U1996 (non-responsive) cells after UNC1999 treatment, and the relative signal quantification. d Western blot against H3 in the INA-6 (responsive) and U1996 (non-responsive) cells after UNC1999 treatment, and the relative signal quantification. The optical density in ( $\mathbf{d}-\mathbf{e})$ was normalized against H4. Statistical analysis in (d-e) was performed with a two-tailed t-test. Values: mean with SEM. Data were collected from three biological replicates. The corresponding uncropped western blot figures (d-e) can be found in Supplementary Fig. 9c. e A principal component analysis (PCA) demonstrating in two dimensions that non-responding and responding samples cluster based on their independent metabolic profiles. $n_{\text {(DMSO INA-6) }}=9, n_{\text {(UNC1999 INA-6) }}=9, n_{\text {(DMSO U1996) }}=11, n_{\text {(UNC1999 U1996) }}=10$. $f$ Heatmap showing the 150 most discriminating metabolites in the dataset, which were significantly different between INA-6 and U1996 after UNC1999 treatment. The colour bar describes the intensity of the change in metabolite levels: deeper colour determines the different magnitude of regulation. Red indicates upregulation and blue indicates downregulation. $n_{\text {(DMSO INA-6) }}=9, n_{\text {(UNC1999 INA-6) }}=9, n_{\text {(DMSO U1996) }}=11, n_{\text {(UNC1999 U1996) }}=10$. g-h Metabolite set enrichment analysis (MSEA) $(\mathbf{g})$ in INA-6 and (h) non-responsive cell lines after UNC1999 treatment. $n_{\text {(DMSO INA-6) }}=9, n_{\text {(UNC1999 INA-6) }}=9$,

$n_{\text {(DMSO KMS-28PE) }}=4, n_{\text {(UNC1999 KMS-28PE) }}=4, n_{\text {(DMSO U266-1970) }}=4, n_{\text {(UNC1999 U266-1970) }}=4, n_{\text {(DMSO u1996) }}=11, n_{\text {(UNC1999 U1996) }}=10 .{ }^{*} p<0.05,{ }^{* *} p<0.01$, ${ }^{* * *} p<0.001,{ }^{* * *} p<0.0001$

Finally, the expression of MTAP was also reduced in INA6 cells after EZH2i (Fig. 3m), in line with the observed accumulation of 5-methylthioadenosine (Fig. 3c). A similar reduction in gene expression was observed in the other responsive cell lines (Supplementary Fig. 3o-w), while no decrease in expression of the above-mentioned genes was observed in the resistant cell line U1996 (Fig. 3e-m). To verify that the changes observed after UNC1999 were due to on-target effects, INA-6 and U1996 cells were treated with a different EZH2i, namely GSK343. A similar gene expression profile and viability effects were found to be induced as with UNC1999 (Supplementary Fig. 4a-l). Altogether, these data suggest that sensitivity to EZH2i was characterised by the downregulation of methionine cycling-associated genes.

\section{Methionine cycling genes were upregulated in MM patients}

Our gene expression analysis suggested that EZH2i impaired the expression of genes involved in methionine cycling in sensitive cell lines. To investigate whether methionine cycling is altered in MM patients and, thus, whether targeting these genes would be of clinical relevance, we performed in silico analysis on patients' gene expression data ${ }^{22}$ (Supplementary Fig. 5a-s). We found that MAT2A, AHCY, MTR and MTAP were increased in monoclonal gammopathy of undetermined significance (MGUS) and smouldering myeloma (SM) as compared to normal plasma cells ${ }^{23}$ (Supplementary Fig. $5 \mathrm{a}, \mathrm{c}, \mathrm{f}$ and $\mathrm{h}$ ). Moreover, MAT2A, MAT2B and $M T R$ were overexpressed in newly diagnosed MM patients as compared to MGUS patients (Supplementary Fig. $5 \mathrm{i}-\mathrm{j}$ and $\mathrm{n}$ ). Finally, the expression of $M A T 2 A$, $M A T 2 B$ and $M T R$ positively correlated with poor prognosis in patients not responding to bortezomib monotherapy ${ }^{24}$ (Supplementary Fig. 5q-s). In summary, methionine cycling-associated genes were found to be overexpressed in MM patients, pointing to them being of clinical relevance.

\section{Downregulation of methionine cycling genes by EZH2i was miRNA-dependent}

To investigate the molecular mechanisms underlying the downregulation of methionine cycling-associated genes in INA-6, we studied whether EZH2i induced expression of miRNAs that could regulate the genes of interest. In silico analysis using miRNA expression data from the INA-6 cell line ${ }^{11}$ identified 306 miRNAs that were upregulated upon UNC1999 treatment, 15 of which were predicted to target methionine cycling-associated genes according to an analysis performed using TargetScanHuman.org ${ }^{25}$ (Supplementary Fig. 6a-g). Of these, six miRNAs were significantly upregulated after UNC1999 treatment (i.e., miR-130a-3p, miR-134-5p, miR-192-5p, miR-4429, miR-223-3p and miR-320c) and four miRNAs showed a trend towards upregulation (i.e., miR-494-3p, miR-23a-3p, miR-21-5p and miR-27a-3p) (Supplementary Fig. $6 \mathrm{~h}-\mathrm{v}$ ). However, only five of these miRNAs (i.e., miR130a-3p, miR-134-5p, miR-192-5p, miR-4429 and miR494-3p) were enriched for H3K27me3 under basal conditions (Supplementary Fig. 7a). These five also exhibited reduced H3K27me3 enrichment in three genomic regions post-UNC1999 treatment (Fig. 4a-e), which was associated with a significant increase in their relative expression (Fig. 4f). In addition, miR-494-3p, miR-130a-3p, miR-134-5p and miR-192-5p also showed reduced EZH2 binding after UNC1999 treatment (Supplementary Fig. $7 b-d)$.

In order to investigate the functional relationship between each miRNA and its predicted target genes (Supplementary Figure 6g), we transfected MCF7 cells with the corresponding miRNA mimics (Supplementary Fig. $7 \mathrm{~g}$ ) and evaluated target gene expression. All five miRNA mimics were detectable 48 -h post-transfection 


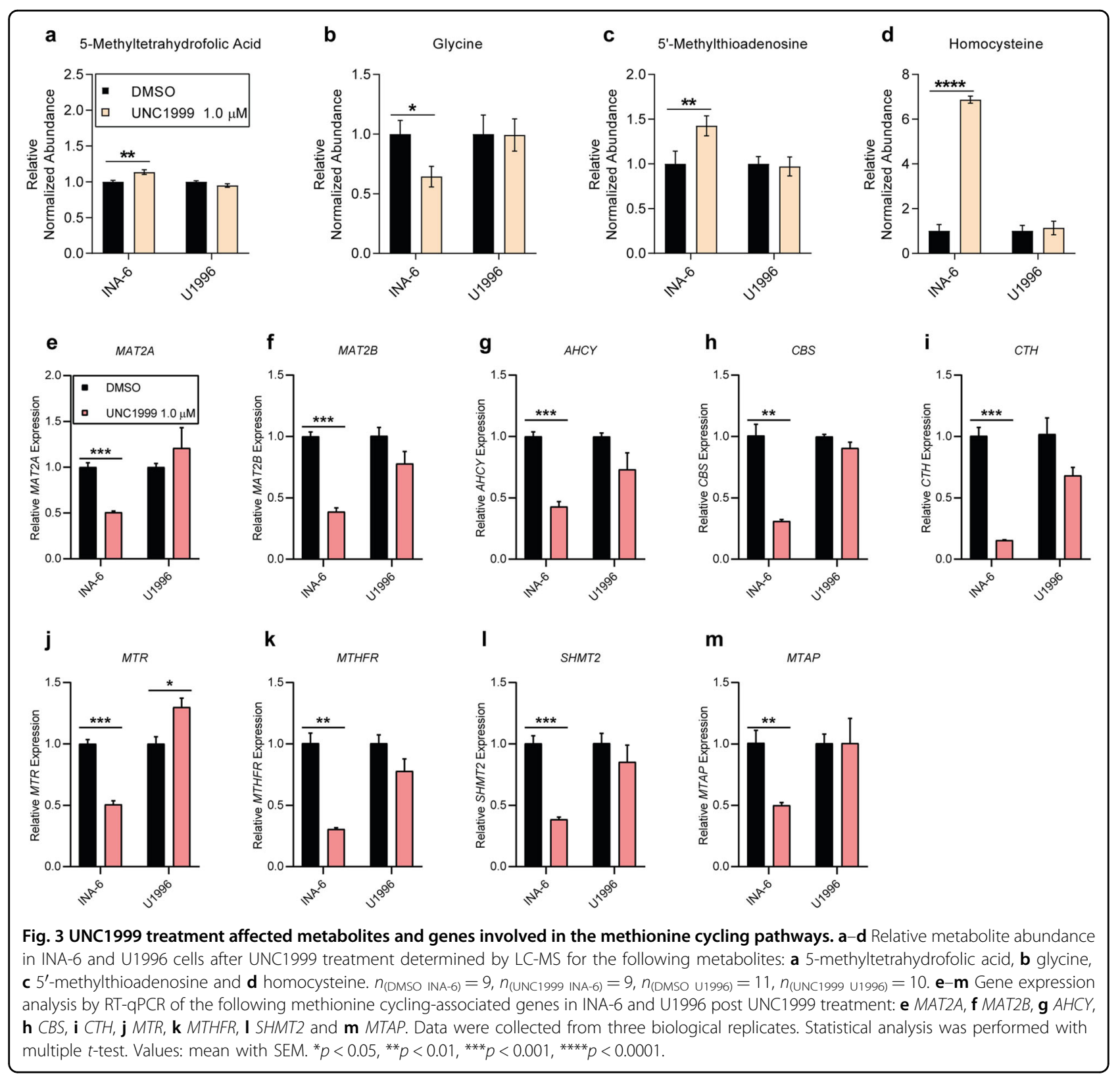

(Supplementary Fig. 7h-l). As predicted, MAT2A was downregulated upon overexpression of miR-494-3p or miR-134-5p, MAT2B expression was reduced upon transfection of miR-134-5p or miR-130a-3p, $C B S$ was downregulated upon overexpression of miR-494-3p and $C T H$ expression was reduced upon transfection with miR192-5p (Fig. 4g-j). MAT2A levels, however, did not decrease upon overexpression of miR-4429 (Fig. 4g). Analysis in the U1996 cell line after UNC1999 treatment did not detect upregulation of any of the five miRNAs (Supplementary Fig. $7 \mathrm{~m}$ ).

Interestingly, DNA methylation was present in $\mathrm{CpG}$ sites surrounding miR-130a-3p and miR-4429 in U1996 cells, but not in INA-6 cells, suggesting that these miRNAs could be silenced by EZH2-independent epigenetic mechanisms in non-responsive cells (Supplementary Fig. 7n). Altogether, we identified five EZH2-targeted miRNAs, which were upregulated in INA-6 cells upon EZH2i and functionally regulated methionine cycling-associated genes.

\section{Sensitivity to EZH2i was associated with DNA damage, G2 arrest and induction of apoptosis}

Accumulation of homocysteine has been associated with an increase in reactive oxygen species (ROS), DNA damage and apoptosis in human leukemic cell lines and 
CBS-deficient leukemic patients ${ }^{26,27}$. Thus, we investigated whether such cytotoxic effects were induced upon UNC1999 treatment in INA-6 cells, which exhibited homocysteine accumulation as a result of treatment (Fig. 3d). Flow cytometry analysis revealed that the reduction in cell viability observed in INA- 6 cells postUNC1999 treatment was accompanied by induction of apoptosis (Fig. 5a and Supplementary Fig. 8a), while U1996 cells remained unaffected (Fig. 5b and Supplementary Fig. 8b). Moreover, enzymes involved in oxidative stress defence (i.e., SOD1 and TRX) were decreased in INA-6 after EZH2i treatment (Supplementary Fig. 8e-f), also in line with increased phosphorylation of histone H2AX ( $\gamma \mathrm{H} 2 \mathrm{AX}$ ) (Fig. 5c, d), a marker for DNA doublestrand breaks. Interestingly, we observed opposite effects in U1996 after EZH2i, i.e., a trend towards increased protection from oxidative stress (Supplementary Fig. $8 \mathrm{e}-\mathrm{f})$ accompanied by a significant reduction of $\gamma \mathrm{H} 2 \mathrm{AX}$ (Fig. 5c), suggesting a functional DNA repair mechanism in the non-responsive cells. In addition, in INA-6 cells UNC1999 triggered the accumulation of cells in G2 phase of the cell cycle, coupled with a reduction of cells entering G1 and S-phase (Fig. 5d and Supplementary Fig. 8c), which was not observed for the U1996 cell line (Fig. 5e and Supplementary Fig. 8d). In summary, sensitivity to EZH2i was associated with accumulation of ROS, DNA damage, apoptosis and G2 arrest.

\section{Discussion}

EZH2, the catalytic subunit of the PRC2 complex, is being extensively investigated as a potential target for cancer therapy and EZH2 inhibitors are in clinical trials for several cancer types ${ }^{28}$. We and others have previously demonstrated that PCR2-mediated gene silencing is a central mechanism for MM tumourigenesis ${ }^{4,10}$ which highlights EZH2 as a potential drug target for MM.

In this study, we evaluated the effect of EZH2i in vivo in the 5T33MM murine model using UNC1999, an EZH2 inhibitor that has shown a safe toxicity profile in mice ${ }^{29}$. EZH2i reduced the tumour burden, emphasising that targeting the PRC2 complex in MM could be of clinical relevance. However, the epigenetic landscape of MM patients is far more heterogeneous. In fact, we have previously investigated a cohort of patient-derived $\mathrm{CD} 138^{+}$ MM cells and found that while UNC1999 induced a strong reduction in cell viability in most samples, several remained viable post-treatment ${ }^{4}$. Moreover, heterogeneous responses to EZH2i in human MM cell lines have been reported in studies using other EZH2 inhibitors ${ }^{30,31}$. Thus, it is of great importance to understand the mechanisms that underlie sensitivity to EZH2i in MM and to find signatures that can discriminate whether a patient is responding to EZH2i.
In diffuse large B-cell lymphoma, resistance to EZH2i can result froThus, we investigated whetherm EZH2 mutations that prevent successful binding of the inhibitor ${ }^{32}$. In MM, however, functional mutations of EZH2 have not yet been observed $^{5}$. The activity of EZH2 depends on multiple metabolites and targeting of EZH2 affects the metabolism of cancer cells ${ }^{33,34}$. Thus, we investigated whether sensitivity and resistance to EZH2i could be discriminated using metabolic profiles. By performing LC-MS analysis, we found that the responsive cell line INA- 6 and the nonresponsive cell line U1996 shared a metabolic profile under basal conditions. Interestingly, however, they exhibited a differential metabolic response upon UNC1999 treatment. Specifically, UNC1999 triggered changes in metabolites involved in methionine cycling in responsive cells, such as accumulation of homocysteine, 5-methyltetrahydrofolic acid, 5-methylthioadenosine and decreased glycine abundance, which were not observed in non-responsive cells. These metabolic alterations may thus represent potential clinical biomarkers of sensitivity to EZH2i. Notably, methods for clinical quantification of 5-methyltetrahydrofolic acid and homocysteine levels in plasma are currently available $^{35,36}$.

As a next step, we sought to uncover the mechanisms underlying the metabolic alterations observed in cells sensitive to EZH2i. EZH2 activity is closely interconnected with the pathways involved in methionine cycling, such as methionine salvage, methionine metabolism, the folate pathway, and the homocysteine degradation pathway (Fig. 6). During methionine metabolism, the enzymes MAT2A/B convert L-methionine to SAM, which by donating a methyl group to EZH2, or other methyltransferases, is converted to SAH. In a second step, SAH is converted to homocysteine by the AHCY enzyme. Homocysteine can then be recycled back to methionine by MTR, which uses 5-methyltetrahydrofolic acid, (5-MTHF), as a methyl donor, or it can be degraded by CBS and CTH within the homocysteine degradation pathway ${ }^{37,38}$.

UNC1999 treatment of sensitive MM cells lines resulted in downregulation of multiple enzymes involved in methionine cycling (including $M A T 2 A$, $M A T 2 B, A H C Y, M T R, C B S, C H T, M T H F R, S H M T 2$ and $M A T P$ ), which is in line with a general shutdown of these pathways and likely underlies the observed metabolic changes. In fact, the downregulation of $C B S$, $C T H$ and MTR suggests that homocysteine and 5MTHF accumulation was due to impaired degradation and re-methylation of homocysteine to methionine. Downregulation of MTHFR, which converts 5,10MTHF to 5-MTHF and of SHMT2, which converts THF into 5,10-MTHF generating the by-product glycine, are consistent with decreased glycine abundance. Finally, downregulation of MATP is consistent with the accumulation of 5-methylthioadenosine (Fig. 6). 


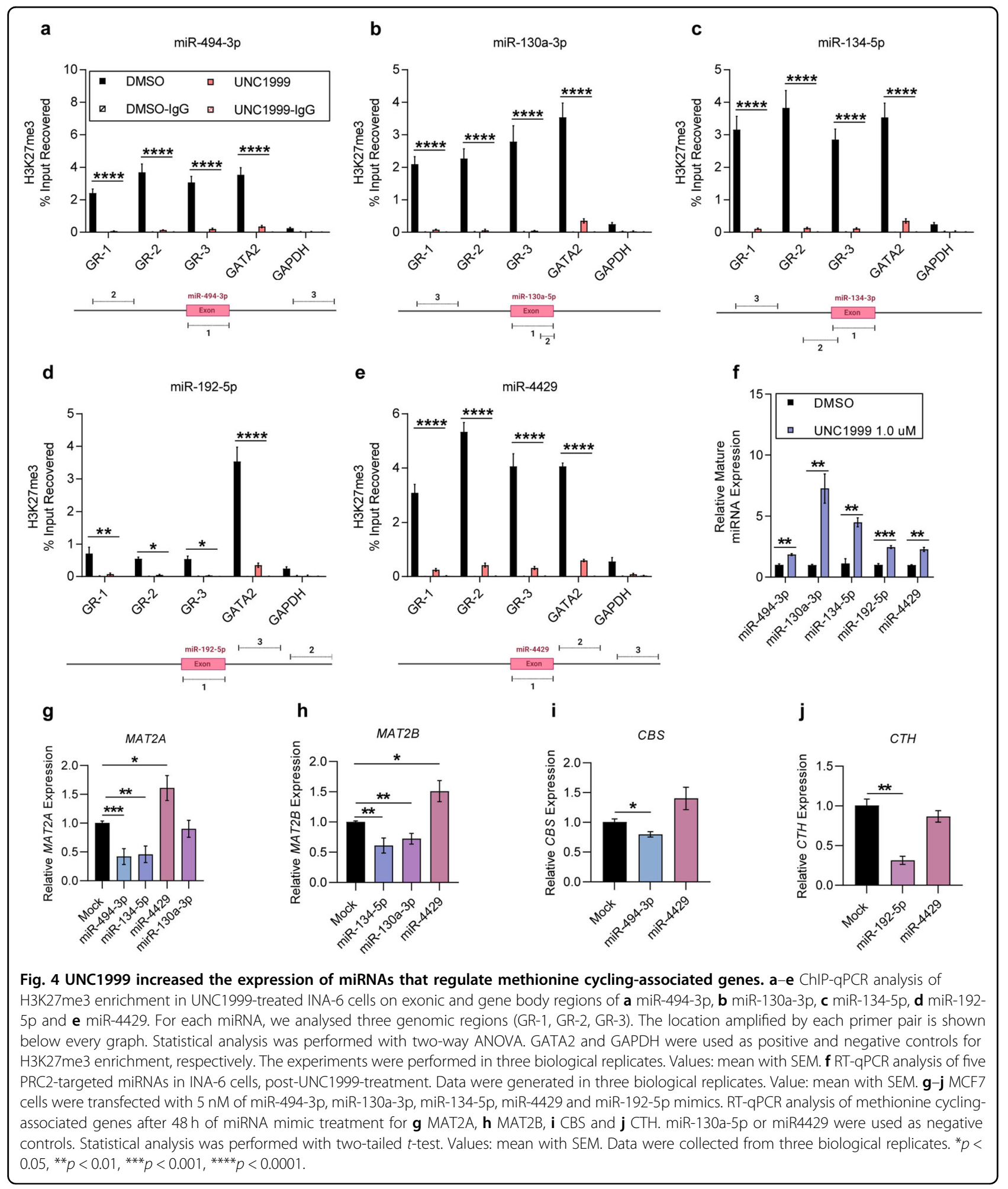

Importantly, we found that some of the above-mentioned genes (i.e., MAT2A, AHCY, MTR and MTAP) were upregulated in MGUS and smouldering myeloma patients as compared to healthy individuals. Moreover, expression of
$M A T 2 A, M A T 2 B$ and $M T R$ increased with disease progression and correlated with poor prognosis in bortezomibresistant patients, suggesting that EZH2i could be beneficial for treatment for MM patients. 

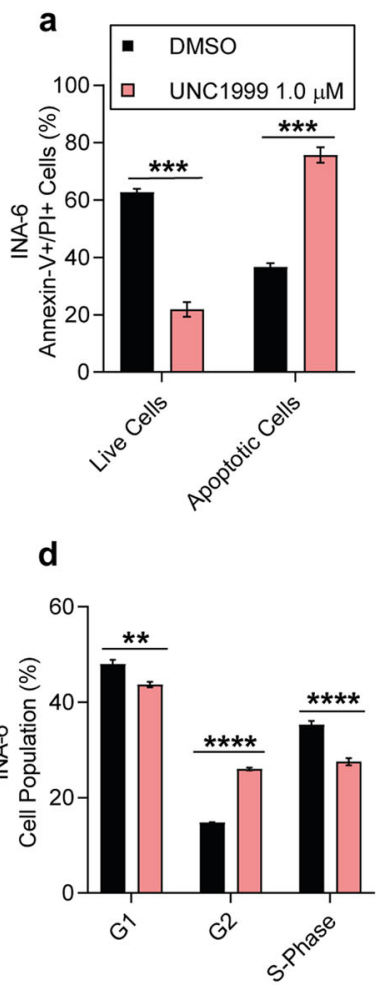

b

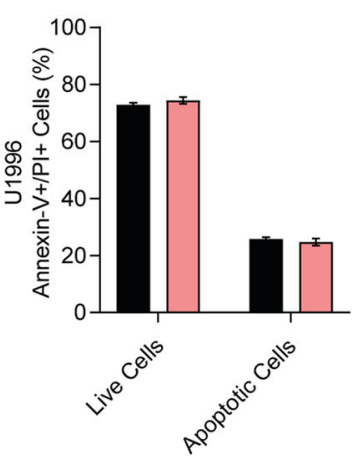

e

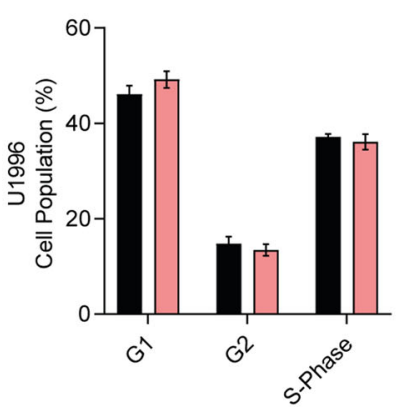

C
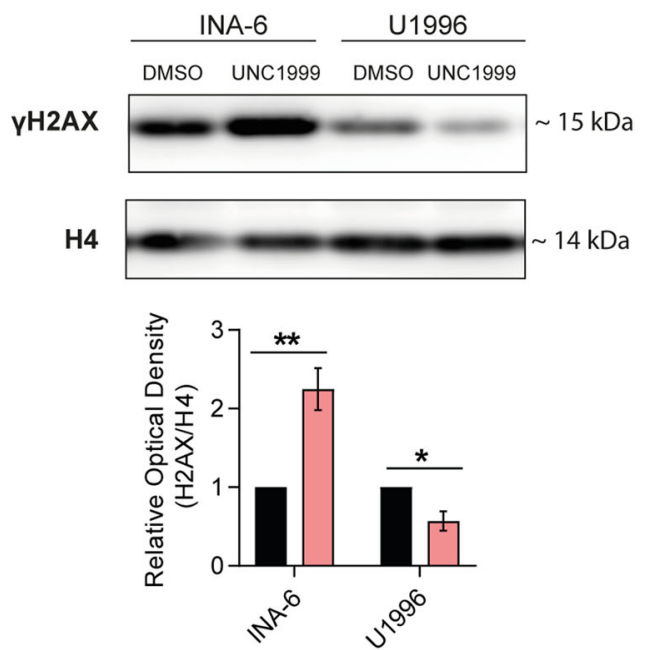

Fig. 5 Sensitivity to EZH2 inhibition was associated with DNA damage, G2 arrest and apoptosis. a-b Flow cytometry analysis of apoptotic markers in (a) INA-6 and (b) U1996 cells upon UNC1999 treatment. c Representative western blot against YH2AX in INA-6 and U1996 cells and signal quantification of the western blot. Optical density was normalized against H4. The corresponding uncropped western blots can be found in Supplementary Fig. 9d. d-e Flow cytometry analysis of the cell cycle in (d) INA-6 and (e) U1996 cells upon UNC1999 treatment. Statistical analysis was performed with one-way ANOVA and multiple t-test. All experiments were performed in three biological replicates. Values: mean with SEM. ${ }^{*} p<0.05$, ${ }^{* *} p<0.01,{ }^{* * *} p<0.001,{ }^{* * *} p<0.0001$.

EZH2 mediates gene silencing by depositing H3K27me3, and its inhibition leads to increased expression of target genes. Thus, it was not directly clear how EZH2i resulted in the downregulation of methionine cycling-associated genes. Increased expression of miRNAs can downregulate target genes and was therefore a plausible mechanism. Consistent with our hypothesis, we found that UNC1999treated cells upregulated four miRNAs (miR-494-3p, miR130a-3p, miR-134-5p and miR-192-5p) that could functionally downregulate methionine cycling-associated genes such as MAT2A, MAT2B, CBS and CTH. Notably, these miRNAs have been reported in the context of several other cancer types $^{39-48}$.

Finally, we investigated how this cascade of events was connected to cell death in sensitive cell lines. Literature shows that the trapping of homocysteine can induce accumulation of ROS, resulting in DNA damage and apoptosis in human leukemic cell lines and CBS-deficient patients $^{26,27}$. Moreover, downregulation of $A H C Y$, which is essential for maintaining cellular methylation ${ }^{49}$, has also been associated with DNA damage and cell cycle arrest ${ }^{50}$. In line with this, sensitive cells exhibited reduced oxidative stress defence, increased DNA double-strand breaks, G2 arrest and induction of apoptosis upon UNC1999 treatment.

In summary, we describe that sensitivity to EZH2i in $\mathrm{MM}$ is associated with a distinct metabolic signature, which results from a miRNA-mediated downregulation of genes involved in methionine cycling. Our study provides new insights into the metabolic response to targeted epigenetic treatment in $\mathrm{MM}$ and suggests that metabolic profiles have the potential as biomarkers for the response to EZH2i in MM.

\section{Materials and methods \\ In vivo studies}

C57BL/KaLwRij female mice were purchased from Envigo (Horst, The Netherlands). Mice were used at 6-10 weeks of age, housed, and treated following the conditions approved by the Ethical Committee for Animal Experiments, VUB (CEP 17-281-3). For evaluation of the effect of short-term UNC1999 treatment on H3K27me3 levels, naive mice were injected with $0.5 \times 10^{6} 5 \mathrm{~T} 33 \mathrm{MM}$ cells at day 0 . At day 14 post-inoculation, mice were 


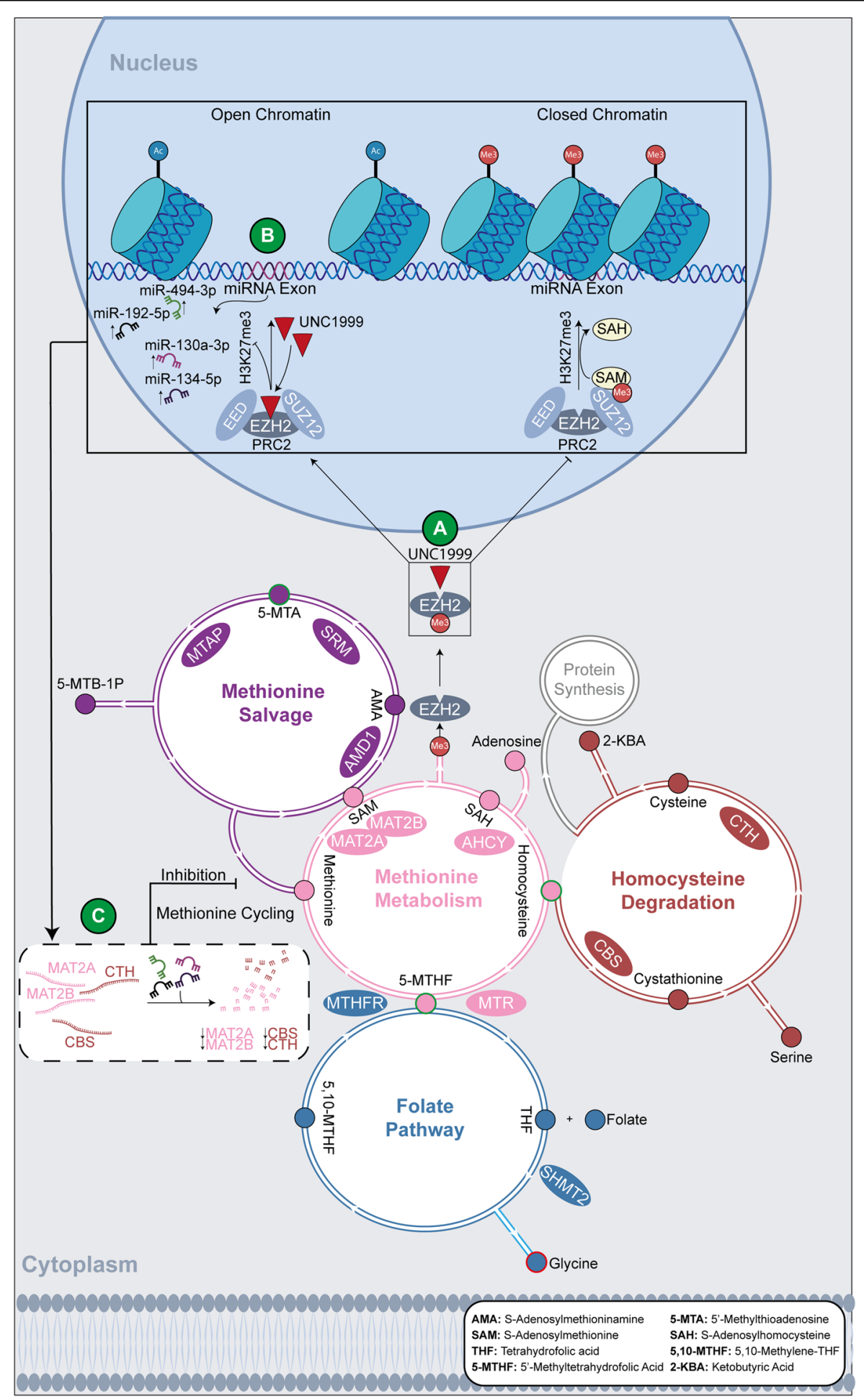

Fig. 6 Schematic representation of the downstream effects of EZH2i on the metabolic profile of sensitive MM cell lines. A UNC1999 inhibits the activity of $\mathrm{EZ} \mathrm{H} 2$. B This prevents $\mathrm{EZH} 2$ from methylating $\mathrm{H} 3$, thus resulting in loss of H3K27me3 and open chromatin. As a consequence, the tumour-suppressor miRNAs miR-494-39, miR-192-5p, miR-130a-3p and miR-134-5p, which are silenced by H3K27me3 under basal conditions, are upregulated. C The tumour-suppressor miRNAs downregulate their target genes (MAT2A, MAT2B, CBS and CTH), which encode for enzymes involved in methionine cycling. This dysregulates methionine cycling and results in variation of metabolite abundance, such as accumulation of homocysteine. Metabolites in each pathway are indicated by small circles, which are colour coded with the same colour of the pathway they participate in. Metabolites that vary in abundance post UNC1999 treatment are marked by either a red (decreased abundance) or a green (accumulation) border. 
randomly assigned to a treatment group receiving 150 or $300 \mathrm{mg} / \mathrm{kg} \mathrm{UNC1999^{29 }}$ daily for seven days $(n=6$; oral gavage), or to a vehicle group receiving $10 \% \mathrm{~N}$-methyl-2pyrrolidinone in PEG300 daily for seven days $(n=6$; oral gavage). We used a predefined value of $n=6$ mice/ treatment. Disease establishment was confirmed by analysis of bone marrow plasmacytosis quantified by May Grünwald-Giemsa staining of bone marrow cytospin samples. The animals were sacrificed when they showed signs of morbidity (paralysis) and the bone marrow harvested after flushing out of the femurs and tibiae and crushing out of the vertebrae. The bone marrow cells were suspended in serum-free medium (RPMI 1640; Cambrex, Europe), supplemented with penicillin-streptomycin, glutamine, and minimal essential medium (MEM) nonessential amino acid (NEAA)-pyruvate (Gibco, Life Technologies) and purified by Lympholyte M (Cedarlane, Hornby, ON, Canada) gradient centrifugation at $1000 \times g$ for $20 \mathrm{~min}$, generating enriched 5T33MM cells. For further enrichment, CD11b + cells were depleted using a MidiMACS magnetic cell separator, LD separation columns and $\mathrm{CD} 11 \mathrm{~b}$ magnetic microbeads (all from Miltenyi Biotec, Bergisch Gladbach, Germany). $5 \mathrm{~T} 33 \mathrm{MMvv}$ cells at a concentration of $1 \times 10^{7}$ cells per $90 \mu \mathrm{l}$ MACS buffer (phosphate-buffered saline containing $0.5 \%$ bovine serum albumin and $2 \mathrm{nM}$ EDTA, $\mathrm{pH} 7.2$ ) and $10 \mu \mathrm{l} \mathrm{CD11b}$ Microbeads were incubated at $4{ }^{\circ} \mathrm{C}$ for $15 \mathrm{~min}$. The 5T33MMvv-microbeads mixture was then loaded onto a separation column placed on the magnetic cell separator and the flow through cell fraction was collected for downstream experiments.

To evaluate the effect of UNC1999 on tumour burden in vivo, 5T33MM cells were injected in C57BL/KalwRij mice as described above. At day 2, mice were randomly assigned to a treatment group receiving $200 \mathrm{mg} / \mathrm{kg}$ UNC1999 every other day ( $n=10$; oral gavage), or to a vehicle group receiving $10 \% \mathrm{~N}$-methyl-2-pyrrolidinone in PEG300 every other day $(n=10$; oral gavage). We performed Wilcoxon-Mann-Whitney power analysis by using GPower $^{51} 3.1$ with a power of 0.9 and an effect size of 2 . Further considering a drop-out rate of $\sim 10 \%$, resulted in 10 mice/ group. The animals were sacrificed when they showed signs of morbidity (paralysis). Tumour load was analysed by means of serum paraprotein concentration quantified by electrophoresis and assessment of total protein; bone marrow plasmacytosis quantified by May Grünwald-Giemsa staining of bone marrow cytospin samples; and spleen weight.

Mice that did not develop the disease or died for other reasons than disease progression were excluded from the analysis.

\section{Cell culture}

The MM cell lines U1996, U266-1970, KMS-28PE, OPM2, L363, LP-1, INA-6 and JJN3 were cultured in
RPMI-1640 AQmediaTM media (Gibco; Thermo Fisher Scientific, Inc., Waltham, MA, USA; cat. no 31870025) with $10 \%$ foetal bovine serum (FBS; Gibco; cat. no 10270106), 1\% GlutaMAX ${ }^{\mathrm{TM}}-\mathrm{I}$ X100 (Gibco; cat. no 35050038) and antibiotics (streptomycin $100 \mu \mathrm{g} / \mathrm{mL}$ and penicillin $100 \mathrm{U} / \mathrm{mL}$; Gibco; cat. no 15140122$)^{52}$. INA-6, U266-1970 and U1996 were supplemented with $10 \mathrm{ng} / \mathrm{mL}$ IL-6 (Prepotech; Cranbury, NJ, USA; cat. no 200-06).

MCF7 cells were cultured in Dulbecco's Modified Eagle Medium/Nutrient Mixture F-12 (DMEM/F-12; Gibco; cat. no 11320033 ) with $10 \%$ FBS (Gibco), $1 \%$ GlutaMAX $^{\mathrm{Tm}}$ I X100 (Gibco) and antibiotics (streptomycin $100 \mu \mathrm{g} / \mathrm{mL}$ and penicillin $100 \mathrm{U} / \mathrm{mL}$; Gibco). All cell lines were cultured at $37^{\circ} \mathrm{C}$ in a humidified $5 \% \mathrm{CO}_{2}$ in-air atmosphere.

For all cell lines, and prior to experiments, we confirmed the absence of Mycoplasma infection by using MycoAlert $^{\mathrm{TM}}$ Mycoplasma Detection Kit (Lonza; Basel, Switzerland; cat. no LT07118) and measurement of luminescence on Lumat LB9597 (Berthold; Bad Wildbad, Germany).

\section{UNC1999 treatment of MM cell lines}

Seven authenticated MM cell lines were selected for UNC1999 drug response analysis ${ }^{29}$. Initially, 100,000 cells $/ \mathrm{mL}$ were seeded in flasks $24 \mathrm{~h}$ prior to the addition of $1 \mu \mathrm{M}$ UNC1999 or DMSO (Sigma-Aldrich; Merck; Darmstadt, Germany; cat. no 317275). The cells received media and reagents change after 3 days. All experiments were performed after 5 days of treatment in cells originating from 3 independent cell batches per cell line.

\section{GSK343 treatment of MM cell lines}

INA-6 and U1996 MM cell lines were selected for drug response analysis to the alternate EZH2 inhibitor GSK343 (Tocris; Bio-Techne; Minneapolis, MN, USA; cat. no 6128). Initially, 100,000 cells $/ \mathrm{mL}$ were seeded in flasks for $24 \mathrm{~h}$ prior to $0.5 \mu \mathrm{M}$ GSK343 or DMSO (Sigma-Aldrich) treatment. The cells received media and reagents change after 3 days. Cells were harvested after 5 days. All experiments were performed in three independent cell batches per cell line.

\section{LC-MS chemicals}

Ammonium formate (LC-MS grade), formic acid (LCMS grade) and methanol (LC-MS grade) were purchased from Sigma-Aldrich; acetonitrile (LC-MS grade) from Fisher Scientific (Zurich, Switzerland) and chloroform (analytical grade) from BDH Laboratory Supplies (Poole, England, UK). The water used was purified using a Milli$\mathrm{Q}^{\mathrm{TM}}$ water system from Millipore (Bedford: MA, USA).

\section{LC-MS sample preparation/metabolite extraction}

For LC-MS, cells were washed in ice-cold PBS and snap frozen. Cell lysis was conducted by two steps of $10 \mathrm{~min}$ of 
thawing-freezing cycles at $37^{\circ} \mathrm{C}$ or in liquid $\mathrm{N}_{2}$, respectively. After, the samples were sonicated at ultrasonic wave output power $320 \mathrm{~W}$ in Bioruptor ${ }^{\circledR}$ (Diagenode; Liège, Belgium) for $30 \mathrm{~s}$ and centrifuged (2000 RCF, $10 \mathrm{~min}, 4^{\circ} \mathrm{C}$ ). The supernatants were transferred to fresh extraction tubes, and a $\mathrm{QC}$ sample was prepared by pooling an equal volume from each sample into a separate extraction tube. Methanol and chloroform were added to the tubes to a final proportion of 2.85:4:4 (water:methanol: choloroform), the tubes gently vortexed and incubated at $6^{\circ} \mathrm{C}$ for $20 \mathrm{~min}$. After centrifugation (2000 RCF, $20 \mathrm{~min}$, $\left.4{ }^{\circ} \mathrm{C}\right)$, the supernatants were transferred to fresh extraction tubes and evaporated at $40^{\circ} \mathrm{C}$ under $\mathrm{N}_{2}(\mathrm{~g})$. The samples were then reconstituted in acetonitrile:water $75: 25^{53-55} n($ INA-6 DMSO $)=9, n($ INA -6 UNC1999 $)=9$; $n(\mathrm{U} 1996 \quad \mathrm{DMSO})=11, \quad n(\mathrm{U} 1996 \quad \mathrm{UNC1999})=10$; $n(\mathrm{KMS}-28 \mathrm{PE}$ DMSO $)=4, n(\mathrm{KMS}-28 \mathrm{PE} \mathrm{UNC1999)}=4$; $n(\mathrm{U} 266-1970 \quad \mathrm{DMSO})=4, \quad n(\mathrm{U} 266-1970 \quad \mathrm{UNC} 1999)$, $n(\mathrm{LP}-1 \quad \mathrm{DMSO})=4, \quad n(\mathrm{LP}-1 \quad \mathrm{UNC} 1999)=4 ; \quad n(\mathrm{~L} 363$ $\mathrm{DMSO})=4, n(\mathrm{~L} 363 \mathrm{UNC} 1999)=4 ; n(\mathrm{OPM} 2 \mathrm{DMSO})=$ 4, $n(\mathrm{OPM} 2 \mathrm{UNC} 1999)=4$.

\section{LC-MS analysis and metabolic profiling}

Reconstituted samples were analysed using an Acquity I-Class UPLC equipped with a BEH Amide column $(2.1 \times$ $50 \mathrm{~mm}, 1.7 \mu \mathrm{m}$ i.d.) hyphenated to a Synapt G2S qTOF through an electrospray ionization (ESI) source, all from Waters (Waters Corporation; Saint-Quentin En Yvelines Cedex, France). MassLynx v. 4.1 (Waters Corporation) was used for instrument control and data collection.

The chromatographic separation was performed at $40{ }^{\circ} \mathrm{C}$ and using $5 \mu \mathrm{L}$ injection volumes. Mobile phase A consisted of 95:5 acetonitrile:water, with $10 \mathrm{mM}$ ammonium formate and $0.1 \%$ formic acid, while mobile phase B consisted of 50:50 acetonitrile:water, with $10 \mathrm{mM}$ ammonium formate and $0.1 \%$ formic acid. The flow rate was set to $0.3 \mathrm{~mL} / \mathrm{min}$, and the gradient started with $100 \%$ for $0.5 \mathrm{~min}$, followed by a non-linear gradient (MassLynx slope factor 8) over $12.5 \mathrm{~min}$ to $100 \% \mathrm{~B}$, then isocratic at $100 \% \mathrm{~B}$ for $4 \mathrm{~min}$, and finally $100 \% \mathrm{~A}$ for $6 \mathrm{~min}$ to reequilibrate the column, i.e., a total runtime of $23 \mathrm{~min}$ per injected sample.

Detection was performed in resolution $\mathrm{MS}^{\mathrm{E}}$ mode, in the range $m / z 50-800$. All samples were analysed in positive mode first, followed by negative mode. The capillary voltage was 1 and $-2 \mathrm{kV}$ and cone voltage 30 and $25 \mathrm{~V}$ for positive and negative mode, respectively. The source temperature was $120^{\circ} \mathrm{C}$ and the de-solvation temperature $500^{\circ} \mathrm{C}$ in both modes. Nitrogen was used as de-solvation and cone gas, at a flow of 800 and $50 \mathrm{~L} / \mathrm{h}$, respectively, in both modes. For $\mathrm{MS}^{\mathrm{E}}$ acquisition, a collision energy ramp from 20 to $45 \mathrm{eV}$ was used, with argon as the collision gas. Lock-mass correction was applied using a solution of leucine-enkephalin.
The QC sample (see sample extraction section) was injected repeatedly before sample analysis to condition the system, to ensure stable analytical conditions, including mass accuracy, instrument sensitivity and chromatographic performance. Furthermore, the QC sample was also injected at regular intervals (every 6th injection) to monitor analytical stability throughout the analysis $^{56}$.

\section{LC-MS data processing}

Data quality was assessed using univariate data analysis of a selected set of metabolites, comprising mass accuracy, retention time and peak area of the QC samples throughout the entire analysis. Raw data files were then converted to NetCDF file format using Databridge (Masslynx version 4.1; Waters S.A.S.). The R-based XCMS package was used for peak detection, retention time alignment and peak grouping ${ }^{57}$.

Briefly, the "centWave" function was used for peak detection in the $0.75-17$ min retention time range, with $10 \mathrm{ppm}$ maximum $\mathrm{m} / \mathrm{z}$ deviation between scans, 5-45 s peak width boundary and signal-to-noise ratio cut-off at five. The "obiwarp" function was used for retention time alignment. For adduct and isotope annotation of the data, R-based package CAMERA was used ${ }^{58}$. The resulting dataset was exported to Microsoft Excel for probabilistic quotient normalization $(\mathrm{PQN})^{59}$. After normalization, all features with a coefficient of normalization $(\mathrm{CV})>30 \%$ in the QC injections were removed ${ }^{56,60,61}$.

\section{LC-MS multivariate and univariate data analysis}

The normalized dataset was imported to Simca P + (version 15, Sartorius Stedim Data Analytics AB, Umeå, Sweden) and pareto scaled. PCA was used to analyse sample groupings, detect outliers and search for systematic trends in the data. Orthogonal projections to latent structures-discriminant analysis (OPLS-DA) was used in combination with shared and unique structures (SUS) plots to pinpoint features related to differences between cell lines as well as treated and control samples $^{62,63}$. OPLS-DA models were built for each set of samples from different cell lines, with treated/control as the discriminating factor. Separate models were built for responsive (INA-6) and non-responsive (U1996) cell lines, respectively. The latter two models were offset using a SUS-plot, and features with $p$ (corr) $\geq 0.4$ were annotated.

For identification, molecular weight, isotopic patterns, fragmentation and, when possible, retention time comparison with an in-house database was used. The Human Metabolome Database (HMDB) and METLIN were used for experimental $\mathrm{m} / \mathrm{z}$ search with a maximum molecular weight difference of $30 \mathrm{ppm}$. All annotated metabolites should be considered putatively annotated (level 2) 
according to the Metabolomics Standards Initiative nomenclature ${ }^{64}$.

Annotated metabolites were subjected to Pathway analysis using MetaboAnalyst $4.0^{21}$. Metaboanalyst was also utilized for heatmap generation of the whole dataset, as well as annotated subsets. One-way analysis of variance (ANOVA) with post hoc Tukey tests were used for significance testing of metabolites, and $p$-values $<0.05$ were considered significant.

\section{RNA extraction, cDNA synthesis and Real-Time quantitative PCR}

RNA was extracted using TRIzol ${ }^{\mathrm{TM}}$ Reagent (Invitrogen; Thermo Fisher Scientific, Inc., cat. no15596018) as described by the manufacturer. Conversion to cDNA was performed by using the Superscript III Reverse Kit Transcriptase (Invitrogen, CA, USA; cat. no 18080044) utilizing $1 \mu \mathrm{g}$ of RNA template. Real-time quantitative PCR analysis was performed with SSOAdvanced Universal SYBR® Green Supermix (Bio-Rad Laboratories, Inc.; CA, USA; cat. no 1725271) using $0.25 \mu \mathrm{M}$ of forward and reverse primers found in Supplementary Table II in three replicates. miRNA reverse transcription was performed using $10 \mathrm{ng}$ of RNA and by utilizing TaqMan® MicroRNA Reverse Transcription Kit (Applied Biosystems; Thermo Fisher Scientific, Inc., cat. no 4366596). TaqMan® gene expression assays were used for miR-4943p, miR-130a-3p, miR-134-5p, miR-192-5p and miR4429, in 3 technical replicates. CFX96 Touch Real-Time PCR Detection System (Bio-Rad Laboratories, Inc.) was used for fluorophore detection. Relative gene and miRNA expression analysis was performed in Bio-Rad CFX Maestro V.1.1 (Bio-Rad Laboratories, Inc.) and calculated with $2^{-\Delta \Delta C T}$ with actin or RNU6B as a reference gene for protein-coding genes and miRNA expression, respectively. All samples were analysed in biological and technical triplicates.

\section{Protein extraction, western blot and image analysis}

MM cell lines, MCF7 cells and 5T33MM bone marrow cells were harvested and washed in ice-cold PBS (Gibco; cat. no 70011044) and collected post centrifugation at $100 \mathrm{RCF}$ for $5 \mathrm{~min}$. Histone proteins were extracted by utilizing a histone extraction kit (Abcam plc.; Cambridge, UK; cat. no 113476) following the manufacturer's instructions. Protein extraction was conducted using RIPA extraction buffer (Millipore; Merck; cat. no 20188) with protease inhibitors (cOmplete $^{\mathrm{TM}}$, EDTA-free Protease Inhibitor Cocktail; Roche; Merck; cat. no 11873580001). Western blot was performed as previously described ${ }^{4}$, using anti-H3K27me3 (1:1000) (Active Motif; Waterloo, Belgium; cat. no 39155), anti- $\gamma \mathrm{H} 2 \mathrm{AX}$ (1:500) (Millipore; Darmstadt, Germany; cat. no 05-636-I), oxidative stress defence cocktail (1:250) (Abcam; cat. no ab179843) and total histone H3 (1:3000) (Abcam; Cambridge, UK; cat. no 1791) antibodies. Total histone H4 (1:1000) (Active Motif; Waterloo, Belgium; cat. no 91295) and actin (1:500) (Santa Cruz Biotechnology, Inc.; Dallas, TX, USA; cat. no sc-47778) were used as loading controls.

Western blot image analysis and quantification was performed in ImageJ V.1.52a $\mathrm{a}^{65}$ and normalized against total histone H4. Cell line western blot quantifications were analysed by multiple t-test correcting for multiple testing by the Holm-Sidak method.

\section{Chromatin immunoprecipitation}

ChIP was performed utilizing the iDeal ChIP-seq kit for histones (Diagenode; Seraing, Belgium; cat. no C01010051) according to the manufacturer's protocol. Chromatin from 7 million cells was crosslinked for $8 \mathrm{~min}$ in 1\% formaldehyde (Thermo Scientific; Thermo Fisher Scientific, Inc., cat. no 28906) at RT, followed by the addition of $0.1 \mathrm{M}$ of glycine to inhibit the crosslinking. The chromatin was sonicated for 10 cycles on Pico Bioruptor ${ }^{\mathrm{TM}}$ (Diagenode) (30 s ON/30 s OFF). For each immunoprecipitation reaction, the chromatin of 200,000 cells was combined with either $5 \mu \mathrm{g}$ anti-H3K27me3 (Active Motif; cat. no 39155), $5 \mu \mathrm{g}$ anti-IgG Rabbit (Millipore, cat. no PP64B), $1 \mu \mathrm{g}$ anti-EZH2 (Millipore, cat. no 17-662) or $1 \mu \mathrm{g}$ anti-IgG Mouse (Millipore; CS200621) antibodies. 10\% of the chromatin used for the IPs was de-crosslinked and purified prior to immunoprecipitation and used as input. qPCR analysis was conducted by SSOAdvanced Universal SYBR ${ }^{\circledR}$ Green Supermix (Bio-Rad Laboratories, Inc.; CA, USA, cat. no 1725271), utilizing $0.25 \mu \mathrm{M}$ of forward and reverse primers found in Supplementary Table II, in two technical replicates. CFX96 Touch Real-Time PCR Detection System (Bio-Rad Laboratories, Inc.) was used for fluorophore detection. Data analysis was performed using \% input $=2^{\wedge}\left[\left(\mathrm{Ct}_{\text {input }}-\log 2^{10}-\mathrm{Ct}_{\text {sample }}\right] \times 100\right.$, as specified by the manufacturer's protocol and plotted against the positive control GATA2 and negative control $\mathrm{GAPDH}^{7}$.

\section{Cell viability assay}

Resazurin reduction protocol was used to evaluate cell viability after UNC1999 treatment ${ }^{66}$. Cells were seeded into a 96-well plate and alamarBlue ${ }^{\mathrm{TM}}$ solution (Sigma-Aldrich, MI, USA, cat. no R7017) was added at $10 \% \mathrm{v} / \mathrm{v}$ in 3 technical replicates. The fluorescent signal was measured by Synergy HTX Plate Reader (BioTek; Winooski, VT, USA) at a threshold of five times the background.

\section{Cell viability staining by flow cytometry}

Cells were stained using the Zombie Aqua $^{\mathrm{TM}}$ Fixable Viability Kit (BioLegend; San Diego, CA, USA; cat. no 423101) following the manufacturer's instructions. The samples were 
analysed on the CytoFLEX LX (Beckman Coulter; Brea, CA, USA). Data were analysed using CytExpert V.2.4.0.28 (Beckman Coulter).

\section{Apoptosis and cell cycle analysis}

Apoptosis assay was performed by harvesting the cells by $210 \mathrm{RCF}$ centrifugation for $5 \mathrm{~min}$. The samples were then washed with PBS and centrifuged for an additional 5 min. The samples were then re-suspended in FITCconjugated Annexin $\mathrm{V}$ and propidium iodine using the TACS Annexin V-FITC Apoptosis Kit (R\&D Systems, Gaithersburg, MD, USA). Cell cycle assay was performed as per the manufacturer's instructions, utilizing BrdU incorporation for $1 \mathrm{~h}$ of sample incubation (559619, BD Pharmingen, CA, USA). All data were collected on the BD LSR Fortessa flow cytometer (BD Bioscience, CA, USA). Data were analysed with Kaluza Analysis flow cytometry software V.1.3 (Beckman Coulter, Brea; CA, USA) and Graphpad Prism V.8.4.3 (Graphpad Software Inc, CA, USA).

\section{MiRNA mimics}

MCF7 cells were seeded at concentration 120,000/well in 6-well plates and allowed to rest for $24 \mathrm{~h}$ prior to transfection. Transfections were performed in at least three biological replicates using RNAiMAX lipofectamine (Invitrogen; cat. no 13778075) according to the manufacturer's instructions and $5 \mathrm{nM}$ of miCURY LNA miRNA mimics (QIAGEN; Supplementary Table IV). Transfection efficiency was evaluated at 24-h post-transfection using $5^{\prime}$ FAM-labbeled negative control mimic on CytoFLEX LX (Beckman Coulter, Brea; CA, USA). Data were analysed using CytExpert V.2.4.0.28 (Beckman Coulter). Detection of mimics and target genes by RT-qPCR was evaluated at 48-h post-transfection.

\section{In silico analysis}

Normalized (MAS5) patient gene expression and survival data (U133 Plus 2.0; Affymetrix, CA, USA) (GEO: GSE2113, GSE9782 and GSE5900) ${ }^{22-24,67}$ were obtained through genomicscape.com. INA-6 miRNA array expression data was obtained from GSE: $87715^{11}$. All data were analysed in Graphpad Prism V.8.4.3 (Graphpad Software Inc, CA, USA). miRNA target prediction was determined by TargetScanHuman ${ }^{25}$.

\section{DNA methylation array}

DNA was extracted from three independent cell batches of the U1996 and the INA-6 MM cell lines using the PureLink ${ }^{\circledR}$ Genomic DNA Mini Kit (Invitrogen, CA, USA) as per manufacturer's protocol. Bisulfite conversion was performed using the EZ DNA Methylation $^{\mathrm{TM}}$ Kit (\#D5004, Zymo Research, CA, USA) with 250 ng of DNA per sample. The bisulfite-converted DNA was eluted in
$15 \mu \mathrm{l}$ according to the manufacturer's protocol, evaporated to a volume of $\langle 4 \mu \mathrm{l}$, and used for methylation analysis by the Illumina Infinium EPIC array ${ }^{68,69}$.

\section{Statistical analysis}

Student $t$-test, multiple $t$-test and one-way ANOVA were utilized to analyse all mice experiments, MGUSMM patient gene expression data and metabolite statistics, respectively. miRNA array data were analysed by student $t$-test. All statistical analyses for apoptosis, cell cycle and ChIP-qPCR were done by two-way ANOVA. Multiple $t$-test was used to analyse RT-qPCR (no correction for multiple testing) and one-way ANOVA was used to analyse normal PC, MGUS and SM patient gene expression and cell viability data. All data were processed in Graphpad Prism V.8.4.3 (Graphpad Software Inc, CA, USA).

\section{Acknowledgements \\ We are grateful to Charlotta Sandberg Blixt for the excellent technical assistance and Pernilla Martinsson for assisting in preparing cells for metabolic profiling. We are grateful to Mohanraj Ramachandran, Alessandra Vaccaro, Tiarne van de Walle and Chuan Jin for their assistance and advice regarding the flow cytometry experiments. Cell cycle/apoptosis flow cytometry analysis was performed at BioVis - Biological Visualization, Science for Life Laboratory, Department of Immunology, Genetics and Pathology, Rudbeck Laboratory, Uppsala University, Sweden. The data handling was enabled by resources provided by the Swedish National Infrastructure for Computing (SNIC) at UPPMAX partially funded by the Swedish Research Council through grant agreement no. 2018-05973.}

\section{Funding statement}

The project was supported by grants from the Swedish Cancer Society (CAN 2016/458), the Hans von Kantzow Foundation, the Swedish Research Council (KDB 1335/17) and the SRP14-VUB. Open Access funding provided by Uppsala University.

\section{Author details}

'Science for Life Laboratory, Department of Immunology, Genetics and Pathology, Rudbeck Laboratory, Uppsala University, Uppsala, Sweden. 2Department of Medicinal Chemistry, Analytical Pharmaceutical Chemistry, Uppsala University, Uppsala, Sweden. ${ }^{3}$ Department of Haematology and Immunology-Myeloma Center Brussels, Vrije Universiteit Brussel (VUB), Brussels, Belgium. ${ }^{4}$ Mount Sinai Center for Therapeutics Discovery, Departments of Pharmacological Sciences and Oncological Sciences, Tisch Cancer Institute, Icahn School of Medicine at Mount Sinai, New York, NY 10029, USA

\section{Author contributions}

P.N., A.A.P., F.Ö., K.V, A.K. and H.J.W. conceptualized the project. P.N., A.A.P., J.H., E.D.B., E.M., B.G.Z., and A.K. acquired data. P.N., A.A.P., J.H., E.D.B., and E.M. performed a formal analysis of the data. P.N., A.A.P., F.Ö., K.V., A.K. and H.J.W. assisted in project investigation. J.J. and A.M. provided reagents. K.V., A.K. and H.J.W. provided acquisition of funding. A.A.P., F.Ö., K.V., A.K. and H.J.W. supervised the project. A.K. and H.J.W. administrated the project. P.N. and J.H. visualized all the data. P.N. organised and integrated the data. P.N. wrote the original manuscript draft. All authors read and approved the final manuscript.

\section{Data availability}

The accession number for DNA methylation array data reported in this paper is GEO:GSE152006

\section{Ethical statement}

Mice were housed and treated following conditions approved by the Ethical Committee for Animal Experiments, VUB (CEP 17-281-3). 


\section{Conflict of interest}

The authors declare that they have no conflict of interest.

\section{Publisher's note}

Springer Nature remains neutral with regard to jurisdictional claims in published maps and institutional affiliations.

Supplementary information The online version contains supplementary material available at https://doi.org/10.1038/s41419-021-03447-8.

Received: 9 July 2020 Revised: 4 January 2021 Accepted: 14 January 2021 Published online: 12 February 2021

\section{References}

1. Manier, S. et al. Genomic complexity of multiple myeloma and its clinical implications. Nat. Rev. Clin. Oncol. 14, 100-113 (2017).

2. Furukawa, Y. \& Kikuchi, J. Molecular pathogenesis of multiple myeloma. Int. J. Clin. Oncol. 20, 413-422 (2015).

3. Walker, B. A. et al. Intraclonal heterogeneity is a critical early event in the development of myeloma and precedes the development of clinical symptoms. Leukemia 28, 384-390 (2014).

4. Agarwal, P. et al. Genome-wide profiling of histone $\mathrm{H} 3$ lysine 27 and lysine 4 trimethylation in multiple myeloma reveals the importance of Polycomb gene targeting and highlights EZH2 as a potential therapeutic target. Oncotarget 7, 6809-6823 (2016).

5. Chapman, M. A. et al. Initial genome sequencing and analysis of multiple myeloma. Nature 471, 467-472 (2011).

6. Walker, B. A. et al. Mutational spectrum, copy number changes, and outcome: results of a sequencing study of patients with newly diagnosed myeloma. J. Clin. Oncol. 33, 3911-3920 (2015).

7. Kalushkova, A. et al. Polycomb target genes are silenced in multiple myeloma. PLOS ONE 5, e11483 (2010).

8. Zhan, F. et al. Global gene expression profiling of multiple myeloma, monoclonal gammopathy of undetermined significance, and normal bone marrow plasma cells. Blood 99, 1745-1757 (2002).

9. Croonquist, P. A. \& Van Ness, B. The polycomb group protein enhancer of zeste homolog $2(E Z H 2)$ is an oncogene that influences myeloma cell growth and the mutant ras phenotype. Oncogene 24, 6269-6280 (2005).

10. Honma, D. et al. Novel orally bioavailable EZH1/2 dual inhibitors with greater antitumor efficacy than an EZH2 selective inhibitor. Cancer Sci. 108, 2069-2078 (2017).

11. Alzrigat, M. et al. EZH2 inhibition in multiple myeloma downregulates myeloma associated oncogenes and upregulates microRNAs with potential tumor suppressor functions. Oncotarget 8, 10213-10224 (2017).

12. Rizq, O. et al. Dual inhibition of EZH2 and EZH1 sensitizes PRC2-dependent tumors to proteasome inhibition. Clin. Cancer Res. 23, 4817-4830 (2017).

13. Rastgoo, N., Pourabdollah, M., Abdi, J., Reece, D. \& Chang, H. Dysregulation of EZH2/miR-138 axis contributes to drug resistance in multiple myeloma by downregulating RBPMS. Leukemia 32, 2471-2482 (2018).

14. Tiziani, $\mathrm{S}$. et al. Metabolimic profiling of drug responses in acute myeloid leukemia cell lines. PLoS ONE 4, e4251 (2009).

15. Lopes-Rodrigues, $V$. et al. Identification of the metabolic alterations associated with the multidrug resistant phenotype in cancer and their intercellular transfer mediated by extracellular vesicles. Sci. Rep. 7, 44541 (2017).

16. Wong, C. C., Qian, Y. \& Yu, J. Interplay between epigenetics and metabolism in oncogenesis: mechanisms and therapeutic approaches. Oncogene 36, 3359-3374 (2017).

17. Vanderkerken, K., Asosingh, K., Croucher, P. \& Van Camp, B. Multiple myeloma biology: lessons from the 5TMM models. Immunol. Rev 194, 196-206 (2003).

18. Asosingh, K., Radl, J., Van Riet, I., Van Camp, B. \& Vanderkerken, K. The 5TMM series: a useful in vivo mouse model of human multiple myeloma. Hematol. J. 1, 351-356 (2000).

19. Zurcher, J. et al. Young C57BL/KaLwRij Mice of the Paraprotein-Producing Clone from Old to Idiopathic Paraproteinemia: II. Transplantation IDIOPATHIC PARAPROTEINEMIA II. Transplantation of the ParaproteinProducing Clone from Old to Young C57BL/KaLwRij Mice. J. Immunol. 122, 609-613 (1979).
20. Vanderkerken, $\mathrm{K}$. et al. Organ involvement and phenotypic adhesion profile of 5T2 and 5T33 myeloma cells in the C57BL/KaLwRij mouse. Br. J. Cancer 76, 451-460 (1997).

21. Chong, J. et al. MetaboAnalyst 4.0: towards more transparent and integrative metabolomics analysis. Nucleic Acids Res 46, W486-W494 (2018).

22. Mattioli, M. et al. Gene expression profiling of plasma cell dyscrasias reveals molecular patterns associated with distinct IGH translocations in multiple myeloma. Oncogene 24, 2461-2473 (2005).

23. Zhan, F. et al. Gene-expression signature of benign monoclonal gammopathy evident in multiple myeloma is linked to good prognosis. Blood 109, 1692-1700 (2007).

24. Mulligan, G. et al. Gene expression profiling and correlation with outcome in clinical trials of the proteasome inhibitor bortezomib. Blood 109, 3177-3188 (2007).

25. Agarwal, V., Bell, G. W., Nam, J. W. \& Bartel, D. P. Predicting effective microRNA target sites in mammalian mRNAs. Elife 4, e05005 (2015).

26. Oikawa, S., Murakami, K. \& Kawanishi, S. Oxidative damage to cellular and isolated DNA by homocysteine: implications for carcinogenesis. Oncogene 22, 3530-3538 (2003).

27. Vanzin, C. S. et al. Homocysteine contribution to DNA damage in cystathionine á-synthase-deficient patients. Gene 539, 270-274 (2014).

28. Duan, R., Du, W. \& Guo, W. EZH2: a novel target for cancer treatment. J. Hematol. Oncol. 13, 104 (2020).

29. Konze, K. D. et al. An orally bioavailable chemical probe of the lysine methyltransferases EZH2 and EZH1. ACS Chem. Biol. 8, 1324-1334 (2013).

30. Hernando, H. et al. EZH2 inhibition blocks multiple myeloma cell growth through upregulation of epithelial tumor suppressor genes. Mol Cancer Ther 15, 287-298 (2016).

31. Ezponda, T. et al. UTX/KDM6A loss enhances the malignant phenotype of multiple myeloma and sensitizes cells to EZH2 inhibition. Cell Rep 21, 628-640 (2017).

32. Bisserier, M. \& Wajapeyee, N. Mechanisms of resistance to ezh2 inhibitors in diffuse large b-cell lymphomas. Blood 131, 2125-2137 (2018).

33. Tao, $\mathrm{T}$. et al. Involvement of $\mathrm{EZH} 2$ in aerobic glycolysis of prostate cancer through miR-181b/HK2 axis. Oncol. Rep 37, 1430-1436 (2017).

34. Ahmad, F. et al. Telomerase reverse transcriptase (TERT) - enhancer of zeste homolog 2 (EZH2) network regulates lipid metabolism and DNA damage responses in glioblastoma. J. Neurochem. 143, 671-683 (2017).

35. Zheng, X. H., Jiang, L. Y., Zhao, L. T., Zhang, Q. Y. \& Ding, L. Simultaneous quantitation of folic acid and 5-methyltetrahydrofolic acid in human plasma by HPLC-MS/MS and its application to a pharmacokinetic study. J. Pharm. Anal. 5, 269-275 (2015).

36. Rosovsky, R. et al. Endothelial stress products and coagulation markers in patients with multiple myeloma treated with lenalidomide plus dexamethasone: an observational study. Br. J. Haematol. 160, 351-358 (2013).

37. Wang, Z. et al. Methionine is a metabolic dependency of tumor-initiating cells. Nat. Med 25, 825-837 (2019).

38. Phillips, C. M. et al. Upregulation of cystathionine- $\beta$-synthase in colonic epithelia reprograms metabolism and promotes carcinogenesis. Cancer Res. 77, 5741-5754 (2017).

39. Salati, S. et al. Deregulated expression of miR-29a-3p, miR-494-3p and miR660-5p affects sensitivity to tyrosine kinase inhibitors in CML leukemic stem cells. Oncotarget 8, 49451-49469 (2017).

40. Tian, C. et al. MicroRNA-494 activation suppresses bone marrow stromal cellmediated drug resistance in acute myeloid leukemia cells. J. Cell Physiol. 232, 1387-1395 (2017)

41. Zhang, $X$. et al. Coordinated silencing of MYC-mediated miR-29 by HDAC3 and $\mathrm{EZH} 2$ as a therapeutic target of histone modification in aggressive B-cell lymphomas. Cancer Cell 22, 506-523 (2012).

42. Zhou, Y. M., Liu, J. \& Sun, W. MiR-130a overcomes gefitinib resistance by targeting met in non-small cell lung cancer cell lines. Asian Pacific J. Cancer Prev 15, 1391-1396 (2014).

43. Fujita, Y. et al. MIR-130a activates apoptotic signaling through activation of caspase-8 in taxane-resistant prostate cancer cells. Prostate 75, 1568-1578 (2015).

44. Sun, H., Fan, G., Deng, C. \& Wu, L. miR-4429 sensitized cervical cancer cells to irradiation by targeting RAD51. J. Cell. Physiol. 23, 185-193 (2020).

45. Chen, K. et al. miR-134 increases the antitumor effects of cytarabine by targeting Mnks in acute myeloid leukemia cells. Onco Targets Ther 11, 3141-3147 (2018). 
46. Tian, C. et al. Low miR-192 expression predicts poor prognosis in pediatric acute myeloid leukemia. Cancer Biomark. 22, 209-215 (2018).

47. Sun, Y., Pan, J., Mao, S. \& Jin, J. IL-17/miR-192/IL-17Rs regulatory feedback loop facilitates multiple myeloma progression. PLoS ONE 9, e114647 (2014).

48. Pichiorri, F. et al. Erratum: Downregulation of p53-inducible microRNAs 192, 194, and 215 Impairs the p53/MDM2 Autoregulatory Loop in Multiple Myeloma Development (Cancer Cell (2010) 18(4) (367-381) (S1535610810003429) (10.1016/j.ccr.2010.09.005)). Cancer Cell. 30, 349-351 (2016).

49. Caudill, M. A. et al. Intracellular S-adenosylhomocysteine concentrations predict global DNA hypomethylation in tissues of methyl-deficient cystathionine beta-synthase heterozygous mice. J. Nutr 131, 2811-2818 (2001).

50. Beluzic, L. et al. Knock-down of AHCY and depletion of adenosine induces DNA damage and cell cycle arrest. Sci. Rep. 8, 14012 (2018).

51. Erdfelder, E., Faul, F., Buchner, A. \& Lang, A. G. Statistical power analyses using G*Power 3.1: Tests for correlation and regression analyses. Behav. Res. Methods 41, 1149-1160 (2009).

52. Jernberg-Wiklund, $\mathrm{H}$. and Nilsson, K. Multiple Myeloma Cell Lines. In: Masters JW and Palsson B, eds Human Cell Culture. New York: Kluwer Academic Publishers; 2002. p. 81-155.

53. León, Z., García-Cañaveras, J. C., Donato, M. T. \& Lahoz, A. Mammalian cell metabolomics: experimental design and sample preparation. Electrophoresis 34, 2762-2775 (2013).

54. Viant, M. R. Revealing the metabolome of animal tissues using $1 \mathrm{H}$ nuclear magnetic resonance spectroscopy. Methods Mol. Biol 358, 229-246 (2007).

55. Teng, Q., Huang, W., Collette, T. W., Ekman, D. R. \& Tan, C. A direct cell quenching method for cell-culture based metabolomics. Metabolomics 5, 199-208 (2009).

56. Engskog, M. K. R., Haglöf, J., Arvidsson, T. \& Pettersson, C. LC-MS based global metabolite profiling: the necessity of high data quality. Metabolomics 12, 114 (2016).

57. Smith, C. A., Want, E. J., O'Maille, G., Abagyan, R. \& Siuzdak, G. XCMS: processing mass spectrometry data for metabolite profiling using nonlinear peak alignment, matching, and identification. Anal. Chem. 78, 779-787 (2006).

58. Kuhl, C., Tautenhahn, R., Böttcher, C., Larson, T. R. \& Neumann, S. CAMERA: an integrated strategy for compound spectra extraction and annotation of liquid chromatography/mass spectrometry data sets. Anal. Chem. 84, 283-289 (2012).

59. Dieterle, F., Ross, A., Schlotterbeck, G. \& Senn, H. Probabilistic quotient normalization as robust method to account for dilution of complex biological mixtures. Application in1H NMR metabonomics. Anal. Chem. 78, 4281-4290 (2006).

60. Want, E. J. et al. Global metabolic profiling procedures for urine using UPLCMS. Nat Protoc. 5, 1005-1018 (2010).

61. Dunn, W. B. et al. Procedures for large-scale metabolic profiling of serum and plasma using gas chromatography and liquid chromatography coupled to mass spectrometry. Nat. Protoc. 6, 1060-1083 (2011).

62. Bylesjö, M. et al. OPLS discriminant analysis: combining the strengths of PLSDA and SIMCA classification. J. Chemom 20, 341-351 (2006).

63. Trygg, J., Holmes, E. \& Lundstedt, T. Chemometrics in metabonomics. J. Proteome Res. 6, 469-479 (2007).

64. Creek, D. J. et al. Metabolite identification: are you sure? And how do your peers gauge your confidence?. Metabolomics 10, 350-353 (2014).

65. Schneider, C. A., Rasband, W. S. \& Eliceiri, K. W. NIH image to ImageJ: 25 years of image analysis. Nat. Methods 9, 671-675 (2012).

66. Walzl, A. et al. The resazurin reduction assay can distinguish cytotoxic from cytostatic compounds in spheroid screening assays. J. Biomol. Screen 19, 1047-1059 (2014).

67. Driscoll, J. J. et al. The sumoylation pathway is dysregulated in multiple myeloma and is associated with adverse patient outcome. Blood $\mathbf{1 1 5}$ 2827-2834 (2010)

68. Gunderson, K. L., Steemers, F. J., Lee, G., Mendoza, L. G. \& Chee, M. S. A genome-wide scalable SNP genotyping assay using microarray technology. Nat. Genet. 37, 549-554 (2005)

69. Steemers, F. J. et al. Whole-genome genotyping with the single-base extension assay. Nat. Methods 3, 31-33 (2006). 